\title{
Muscimol microinjection into cerebellar fastigial nucleus exacerbates stress-induced gastric mucosal damage in rats
}

\author{
Jin-zhou ZHU ${ }^{1,2, \#}$, Su-juan FEI ${ }^{1, \#}$, Jian-fu ZHANG ${ }^{1,2, ~ *}$, Sheng-ping ZHU ${ }^{1,2}$, Zhang-bo LIU ${ }^{1,2}$, Ting-ting LI, ${ }^{1,2}$, Xiao QIAO ${ }^{1,2}$ \\ ${ }^{1}$ Department of Gastroenterology, Affiliated Hospital of Xuzhou Medical College, Xuzhou 221002, China; ${ }^{2}$ Department of Physiology, \\ Xuzhou Medical College, Xuzhou 221002, China
}

\begin{abstract}
Aim: To investigate the effects of microinjection of the GABA receptor agonist muscimol into cerebellar fastigial nucleus (FN) on stressinduced gastric mucosal damage and the underlying mechanism in rats.

Methods: Stress-induced gastric mucosal damage was induced in adult male SD rats by restraining and immersing them in cold water for $3 \mathrm{~h}$. GABA $\mathrm{A}$ receptor agonist or antagonist was microinjected into the lateral FN. The decussation of superior cerebellar peduncle (DSCP) was electrically destroyed and the lateral hypothalamic area (LHA) was chemically ablated by microinjection of kainic acid. The pathological changes in the gastric mucosa were evaluated using TUNEL staining, immunohistochemistry staining and Western blotting.

Results: Microinjection of muscimol (1.25, 2.5, and $5.0 \mu \mathrm{g})$ into FN significantly exacerbated the stress-induced gastric mucosal damage in a dose-dependent manner, whereas microinjection of $\mathrm{GABA}_{\mathrm{A}}$ receptor antagonist bicuculline attenuated the damage. The intensifying effect of muscimol on gastric mucosal damage was abolished by electrical lesion of DSCP or chemical ablation of LHA performed $3 \mathrm{~d}$ before microinjection of muscimol. Microinjection of muscimol markedly increased the discharge frequency of the greater splanchnic nerve, significantly increased the gastric acid volume and acidity, and further reduced the gastric mucosal blood flow. In the gastric mucosa, further reduced proliferation cells, enhanced apoptosis, and decreased anti-oxidant levels were observed following microinjection of muscimol.

Conclusion: Cerebellar FN participates in the regulation of stress-induced gastric mucosal damage, and cerebello-hypothalamic circuits contribute to the process.
\end{abstract}

Keywords: cerebellum; fastigial nucleus; decussation of superior cerebellar peduncle; lateral hypothalamic area; GABA; muscimol; bicuculline; gastric mucosa; stress-induced gastric mucosal damage; apoptosis

Acta Pharmacologica Sinica (2013) 34: 205-213; doi: 10.1038/aps.2012.152; published online 17 Dec 2012

\section{Introduction}

In recent years, increasing evidence has indicated that the development of stress-induced gastric mucosal damage is associated with variations in the gastrointestinal tract, including gastrointestinal function, gastric mucosal blood flow, and gastric epithelial cells ${ }^{[1,2]}$. However, we know little about the role of the central nervous system (CNS) in regulating stressinduced gastric mucosal damage.

In a previous study, we discovered that stimulation of the fastigial nucleus (FN) in the cerebellum markedly attenuated the stress-induced gastric mucosal damage ${ }^{[3]}$. In contrast,

\footnotetext{
\# These authors contributed equally to this article.

* To whom correspondence should be addressed.

E-mail jfzhang@xzmc.edu.cn

Received 2012-05-15 Accepted 2012-09-29
}

chemical ablation of FN exacerbated this damage. However, the details of the mechanism underlying these processes are unclear.

Numerous auto-radiographic and immunocytochemical studies have demonstrated that the somata and axon terminals of Purkinje cells contain gamma-aminobutyric acid (GABA) ${ }^{[4]}$. Meanwhile, deep cerebellar nuclear neurons are primarily controlled via GABAergic neuron-mediated inhibitory input from Purkinje cells in the cerebellar cortex ${ }^{[5-8]}$. GABA Gecep- $^{-}$ tors were confirmed to be present in the $\mathrm{FN}$ and to receive Purkinje cell projections ${ }^{[9-11]}$. Thus, we suspected that GABA $_{A}$ receptors in the $\mathrm{FN}$ are potentially involved in the regulation of the cerebellum in stress-induced gastric mucosal damage.

Recently, direct bidirectional connections were revealed to exist between the cerebellum and hypothalamus: ie, these are the hypothalamic-cerebellar projection and the 
cerebellar-hypothalamic projection, which constitute the cerebellar-hypothalamic circuits ${ }^{[12]}$. The cerebellar-hypothalamic projection arises from the $\mathrm{DCN}$, passes through the decussation of superior cerebellar peduncle (DSCP) and projects into the lateral hypothalamic area (LHA). In the present research, the role of cerebellar-hypothalamic circuits in the regulation of the FN on stress-induced gastric mucosal damage will be discussed.

LHA has been demonstrated to be linked to celiac sympathetic and parasympathetic nerves, which constitute the greater splanchnic nerve $(\mathrm{GSN})^{[13]}$. Our previous studies showed that variations in GSN discharge frequency are associated with the regulation of stomach functions ${ }^{[14,15]}$. Consequently, studying the effect of changes in the GSN on this regulation is essential.

The gastric mucosa is maintained by a balance between the apoptosis and the proliferation of mucosal epithelium cells ${ }^{[16]}$. Numerous reports have suggested that gastric gland secretory function and oxidation contribute to stress-induced gastric mucosal damage ${ }^{[1,2]}$. Such pathological changes may break down the system that protects gastric mucosa cells, resulting in an erosion or ulcer as a result of attacks by acid or pepsin. Therefore, revealing how the FN regulates stress-induced gastric damage could help treat various gastrointestinal diseases.

\section{Materials and methods Animals}

All procedures involving animals were approved by the Animal Welfare Committee of Xuzhou Medical College, and all experiments were conducted in accordance with the USA National Institute of Health Guide for the Care and Use of Laboratory Animals. Adult male Sprague-Dawley rats, weighing 200-230 g, were supplied by the Experimental Animal Center of Xuzhou Medical College, Xuzhou, China (Usage Certificate No: SYXK [SU] 2002-0038). The rats in each group were housed in wire mesh cages with food and water available at random and were adapted to laboratory conditions. The room temperature was maintained at $23 \pm 1{ }^{\circ} \mathrm{C}$ under a $12 / 12 \mathrm{~h}$ day/night period cycle. Prior to the induction of restraint and water $\left(21 \pm 1^{\circ} \mathrm{C}\right)$-immersion (RWI), all rats were fasted for $24 \mathrm{~h}$ but allowed free access to tap water.

\section{Reagents}

Muscimol, bicuculline and kainic acid were obtained from Sigma-Aldrich (St Louis, USA). The In Situ Apoptosis Detection Kit, POD was purchased from Roche Diagnostics (Berlin, Germany). 3,3'-Diaminobenzidine (DAB), rabbit anti-Caspase-3, rabbit anti-Bcl-2, rabbit anti-Bax, rabbit anti- $\beta$-actin and rabbit anti-proliferating cell nuclear antigen (PCNA) antibodies and alkaline phosphorylase-tagged goat anti-rabbit IgG antibody were obtained from Zhongshan Golden Bridge Biotech Co (Beijing, China). The SABC rabbit IgG POD kit and BCIP/ NBT assay kit were purchased from Boster Bio-engineering (Wuhan, China). The malondialdehyde (MDA) and superoxide dismutase (SOD) assay kits were obtained from Jiancheng Bioengineering (Nanjing, China).

\section{Orientation of brain nuclei}

The rats were anesthetized with sodium pentobarbital hypodermically $(0.04 \mathrm{~g} / \mathrm{kg})$ and subsequently mounted on a stereotactic apparatus. The scalp was incised, and a hole, 0.5 $\mathrm{mm}$ in diameter, was drilled in the cranium, dorsally to the target site. The coordinates for the locations of the FN, DSCP and LHA were determined according to the rat brain atlas in stereotaxic coordinates ${ }^{[17]}$ as follows: AP $11.6 \mathrm{~mm}$, LR $1.0 \mathrm{~mm}$, H $5.6 \mathrm{~mm}$; AP $7.4 \mathrm{~mm}$, LR $0 \mathrm{~mm}, \mathrm{H}$ 7.8-8.0 mm; and AP 2.8 $\mathrm{mm}$, LR $1.5 \mathrm{~mm}, \mathrm{H} 8.3-8.5 \mathrm{~mm}$, respectively. The incisor bar was positioned $3.3 \mathrm{~mm}$ below the center of the aural bar. To confirm that all the brain sites were microinjected or destroyed correctly, after the stomachs had been removed at the end of the experiment, the rats were perfused intracardially with $4 \%$ neutral formaldehyde, and then the brains were extracted and immersed in 10\% paraformaldehyde before fixation for $48 \mathrm{~h}$. The brains were later frozen-sectioned and stained with $1 \%$ neutral red to confirm the sites of the lesions and microinjections. Data from those rats with target sites that were not in accord with the histological criteria were excluded from the statistical analysis.

\section{Microinjection and electrical destruction}

Muscimol, a $\mathrm{GABA}_{\mathrm{A}}$ receptor agonist, was microinjected into the bilateral $\mathrm{FN}$ via a cannula connected to a microsyringe with a polyethylene tube. The regular dose of muscimol was $2.50 \mu \mathrm{g}$ in a volume of $0.3 \mu \mathrm{L} 0.9 \%$ saline in all the muscimol injection groups. However, we used three different doses $(1.25,2.50 \text {, and } 5.00 \mu \mathrm{g} \text { in a volume of } 0.3 \mu \mathrm{L} 0.9 \% \text { saline })^{[18]}$ when we studied the relationship between the dose and the effect. The injection lasted for $2 \mathrm{~min}$, and the injection cannula was left in place for another $3 \mathrm{~min}$ to prevent backflow. Similarly, the vehicle $(0.3 \mu \mathrm{L} 0.9 \%$ saline $)$ and bicuculline (5.00 $\mu \mathrm{g}$ in $0.3 \mu \mathrm{L} 0.9 \%$ saline), a $\mathrm{GABA}_{\mathrm{A}}$ receptor antagonist, were each microinjected into the FN. The electrical ablation of the $\mathrm{DSCP}^{[3]}$ was performed using a positive DC current of $1 \mathrm{~mA}$ for $10 \mathrm{~s}$. The sham DSCP electrical ablation group was treated with the same procedure as above but without any current. The chemical ablation of the LHA was conducted by the microinjection of kainic acid $(0.30 \mu \mathrm{g}$ in a volume of $0.3 \mu \mathrm{L}$ saline $)^{[19]}$ into the bilateral LHA. Saline was microinjected in the sham LHA ablation group. In the experiments, the DSCP and LHA were destroyed electrically or chemically $3 \mathrm{~d}$ before the microinjection of muscimol $(2.50 \mu \mathrm{g}$ in a volume of $0.3 \mu \mathrm{L}$ $0.9 \%$ saline) and RWI.

\section{Model preparation}

In the experimental animals, the FN was microinjected $30 \mathrm{~min}$ prior to the induction of stress-induced gastric mucosal damage. The animals were then lightly anesthetized with ether and fixed to a lab-made framework. The model of stressinduced gastric mucosal damage was prepared according to a previously described method ${ }^{[20]}$. The rats were restrained and immersed in cold water $\left(21 \pm 1^{\circ} \mathrm{C}\right)$ for $3 \mathrm{~h}$ to develop the stress model. 


\section{Assessment of the gastric mucosal damage index}

The gastric mucosal damage index (GMDI) was measured as described ${ }^{[21]}$. At the end of the experiments, the rats were anesthetized with an overdose of sodium pentobarbital. The stomach was incised along the greater curvature and flushed with cold PBS $(0.1 \mathrm{~mol} / \mathrm{L})$. The index was based on a cumulative-length scale on which an individual lesion limited to the mucosal epithelium was scored according to its length as follows: 1, $\leq 1 \mathrm{~mm} ; 2,>1 \mathrm{~mm}$ and $\leq 2 \mathrm{~mm}$; and 3, > $2 \mathrm{~mm}$ and $\leq 3$ $\mathrm{mm}$. For those lesions $>1 \mathrm{~mm}$ in width, the lesion score was doubled. The summation of the scores of all lesions represents the GMDI, which was determined by a researcher who was blinded to the treatments to avoid bias.

\section{Measurement of discharge frequency of greater splanchnic} nerve

The discharge frequency of the GSN was measured as described $^{[22]}$. It was recorded for $2 \mathrm{~min}$ by single barrel electrodes, wrapped with glass, before and after the microinjection of muscimol into the FN. The discharge signals were amplified and filtered via a preamplifier and stored in a computer equipped with the MedLab-U/4C501 biological signal acquisition system (Nanjing Mei-yi Science and Technology Co, Ltd, Nanjing, China), which automatically examined the discharge frequency of the GSN. Thus, only the GSN activities in the stable discharge were recorded.

\section{Measurement of gastric mucosal blood flow}

The measurement of gastric mucosal blood flow was performed as previously described ${ }^{[3]}$. It was measured with a Laser-Doppler flowmeter (LDF-2, Nankai University, Tianjin, China). Briefly, the rats were anesthetized with sodium pentobarbital $(40 \mathrm{mg} / \mathrm{kg}$ ), the abdomen was opened, the stomach was exposed and transected, and the gastric content was slightly evacuated to the exterior through the cut $(5 \mathrm{~mm})$ in the stomach. The laser probe was placed $0.5 \mathrm{~mm}$ above and perpendicular to the mucosal surface to observe the gastric mucosal blood flow displayed on the digital panel of the flowmeter. When the blood flow was stable, four points were selected to record (one point for $1 \mathrm{~min}$ ), and then the average value was calculated.

\section{Immunohistochemistry staining assay}

At the end of the experiments, the rats were anesthetized with an overdose of sodium pentobarbital. The stomachs were rapidly removed and opened along the greater curvature. One half was fixed in $10 \%$ paraformaldehyde for $48 \mathrm{~h}$, sliced into $4 \mu \mathrm{m}$ thick sections and mounted on glass slides for immunohistochemical staining and transferase-mediated dUTPbiotin nick end-labeling (TUNEL) assay. The assessment of gastric mucosal cell proliferation was determined by the SABC immunohistochemical method as previously described ${ }^{[23]}$. We chose rabbit anti-PCNA (diluted to 1:100 in PBS) and biotinylated goat anti-rabbit IgG antibody as the primary and bridging antibodies. The primary antibody was replaced by PBS for the negative control. The quantitative analysis was performed blindly by counting the number of PCNA-positive cells (the presence of brown granules in the nucleus defined a positive cell) and the total cell number in ten random microscopic fields. The proliferation percentage is the percentage of proliferative cells (positive cells/total cells $\times 100 \%$ ) in each group calculated from three inconsecutive sections selected from each specimen.

\section{TUNEL assay}

The TUNEL staining was performed on paraffin sections with an in situ cell death detection kit, according to the manufacturer's instructions and as described in a previous work ${ }^{[24]}$. Hematoxylin was used to counterstain the sections. The average number of TUNEL-positive cells in each group was calculated from three inconsecutive sections selected from each specimen. Finally, a quantitative analysis was performed by counting the number of TUNEL-positive cells in ten random microscopic fields.

\section{Western blotting}

At the end of the experiments, the other half of the gastric mucosa was stored at $-80^{\circ} \mathrm{C}$ for Western blotting. The process was performed as previously reported ${ }^{[25]}$. The protein concentration was determined using a BCA protein assay kit. The sample protein was separated by $10 \%$ SDS-polyacrylamide gel electrophoresis and subsequently transferred to a nitrocellulose membrane. The membranes were immunoblotted with primary antibodies that recognize MAPKs (anti-bax antibody 1:500, anti-Bcl-2 antibody 1:500, anti-Caspase-3 antibody 1:750 and anti- $\beta$-actin antibody 1:2000). The detection was performed with alkaline phosphatase goat anti-rabbit IgG (1:1000) and then developed with the BCIP/NBT assay kit. Then, the bands were scanned and analyzed by Image-Pro plus 6.0.

\section{Measurement of malondialdehyde content and superoxide dismutase activity}

The measurement of MDA and SOD was performed as described $^{[26]}$. To detect the MDA content and SOD activity, the gastric mucosa was stored at $4{ }^{\circ} \mathrm{C}$. The homogenate had been centrifuged at $3000 \times g$ for $10 \mathrm{~min}$, and the supernatant was retained. The protein concentration was determined by the Coomassie brilliant blue protein assay. The level of lipid peroxidation was detected by thiobarbituric acid-reactive substances and detected spectrophotometrically at $532 \mathrm{~nm}$. The SOD activity was detected spectrophotometrically at $550 \mathrm{~nm}$ by the xanthine/xanthine oxidase reaction method. The MDA content and SOD activity were expressed in nmol/mg and $\mathrm{U} / \mathrm{mg}$ individually.

\section{Measurement of gastric juice volume and acidity}

The measurement of the gastric juice volume and acidity was performed as previously described ${ }^{[27]}$. After the microinjection of muscimol into the cerebellar $\mathrm{FN}$, the rat abdomen was incised along the midline. A polyethylene tube was inserted in the low side of the stomach while the pylorus was ligated, and then the abdominal incision was sutured. The gastric 
fluid was collected for $3 \mathrm{~h}$. Based on the results, we detected the variation in the gastric acid secretory functions.

\section{Statistical analysis}

All results are expressed as the mean \pm SEM. Comparisons between two groups were conducted using Student's $t$-test, and multiple-group analyses were conducted by the one-way ANOVA test. The statistical analyses were performed using GraphPad Prism 5 and SPSS 16.0. The results were considered to be significantly different at $P<0.05$.

\section{Results}

\section{Histological verification}

The histological verification was performed on all target sites of electrical and chemical lesions and of microinjections by referring to the stereotaxic atlas (Figure 1).

Effect of muscimol and bicuculline microinjection into the FN on stress-induced gastric mucosal damage

The GMDI was $54.33 \pm 3.47$ in the RWI group and $53.67 \pm 3.45$ in Vehicle group; in contrast, no damage was observed in the
Normal group. Compared with the Vehicle group, the GMDI was obviously increased $(75.17 \pm 3.55,88.17 \pm 3.95,109.2 \pm 4.16$, $n=6, P<0.01)$ following the microinjection of different doses of muscimol into the FN (Figure 2A). Statistical analysis of the correlation between the doses and the GMDI indicated that the correlation was significant at the 0.01 level. Additionally, bicuculline microinjection into the FN reduced the GMDI (27.83 $\pm 4.06, P<0.01$ vs vehicle).

\section{Role of the decussation of superior cerebellar peduncle (DSCP)}

To further ascertain whether the DSCP helps mediate this process, electrical ablation of the DSCP was executed $3 \mathrm{~d}$ prior to the microinjection and RWI. The result showed that the GMDI was $87.33 \pm 2.23$ in the Sham DSCP Electrical Ablation+muscimol+RWI group and was lower, 55.83 \pm 2.75 , in the DSCP Electrical Ablation+muscimol+RWI group. This comparison showed the difference between the two groups was significant $(P<0.01$, Figure $2 \mathrm{~B})$.

\section{Role of the lateral hypothalamic area (LHA)}

To investigate whether the intensifying effect of the muscimol
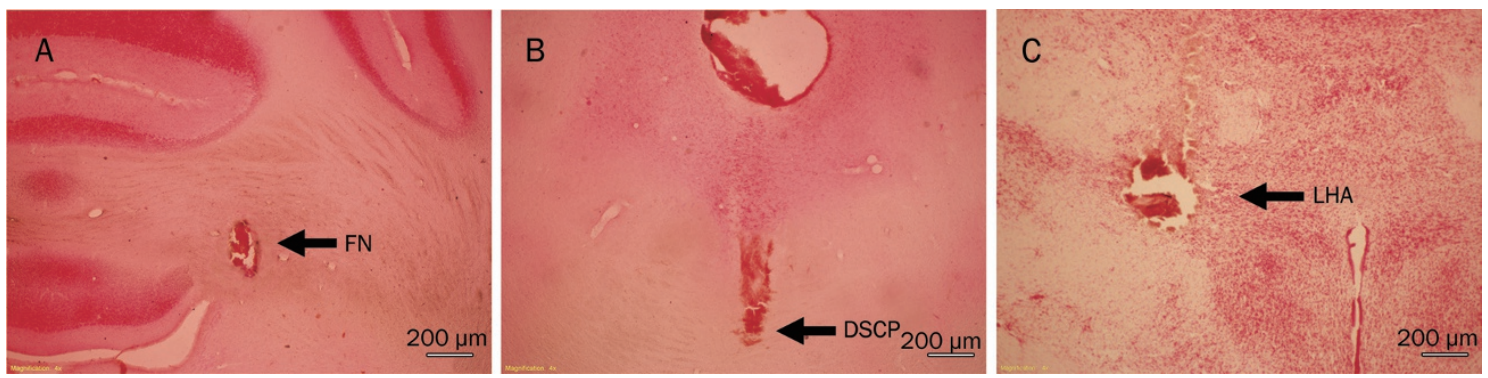

Figure 1. The target sites in the rats' brains. (A) Photomicrograph of microinjection site of FN; (B and C) photomicrographs of ablation sites of DSCP and LHA in the rats' brains. The sections were stained with neutral red, showing the positions (scale bar: $200 \mu \mathrm{m}$ ).
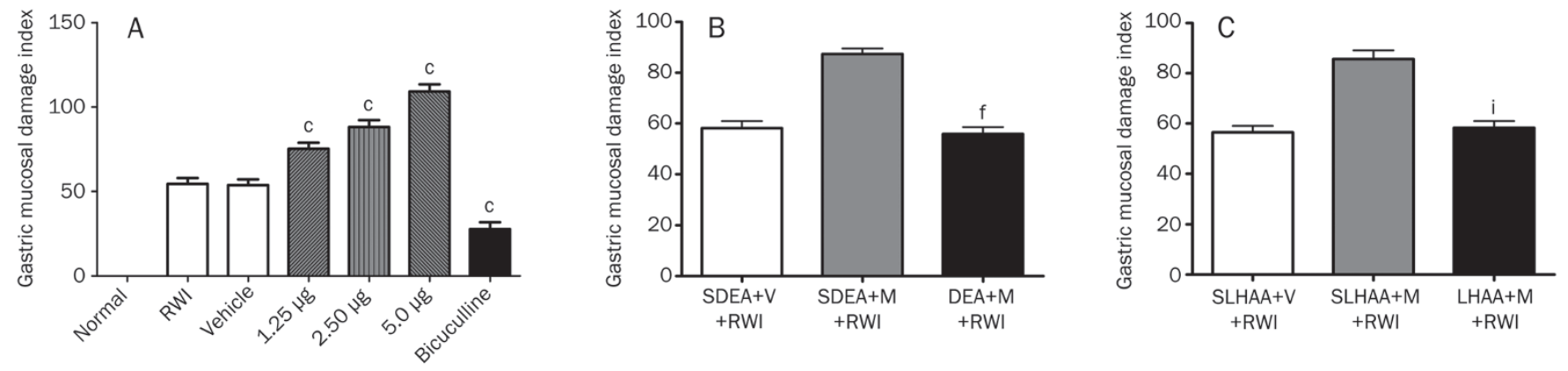

Figure 2. The roles of FN, DSCP, and LHA on stress-induced gastric mucosal damage in rats. (A) Effects of microinjections of muscimol and bicuculline into FN on stress-induced gastric mucosal damage in rats. Normal: normal; RWI: restraint and water $\left(21 \pm 1^{\circ} \mathrm{C}\right)$-immersion, only; Vehicle: microinjection of Vehicle into FN+RWI; $1.25 \mu \mathrm{g}, 2.50 \mu \mathrm{g}, 5.00 \mu \mathrm{g}$ : different doses of muscimol were microinjected into the FN individually+RWI; bicuculline: bicuculline microinjection into FN+RWI. Mean \pm SEM. $n=6$. ${ }^{\circ} P<0.01$ vs Vehicle group. (B) Role of DSCP in muscimol microinjection into FN on stressinduced gastric mucosal damage in rats. SDEA+V+RWI: sham DSCP electrical ablation+microinjection of Vehicle into FN+RWI; SDEA+M+RWI: sham DSCP electrical ablation+microinjection of muscimol into FN+RWI; DEA+M+RWI: DSCP electrical ablation+microinjection of muscimol into FN+RWI. Mean \pm SEM. $n=6$. ${ }^{\mathrm{f}} P<0.01$ vs SDEA+M+RWI group. (C) Role of LHA in muscimol microinjection into FN on stress-induced gastric mucosal damage in rats. SLHAA+V+RWI: sham LHA ablation+microinjection of Vehicle into FN+RWI; SLHAA+M+RWI: sham LHA ablation+microinjection of muscimol into FN+RWI; LHAA+M+RWI: LHA ablation+microinjection of muscimol into FN+RWI. Mean \pm SEM. $n=6$. ' $P<0.01$ vs SLHAA+M+RWI group. 
microinjection into the $\mathrm{FN}$ is mediated through the LHA, the LHA was damaged $3 \mathrm{~d}$ before the microinjection of muscimol into the FN. The GMDI was $85.67 \pm 3.48$ in the Sham LHA Ablation+muscimol+RWI group and was lower, 58.33 \pm 2.64 , in the LHA Ablation+muscimol+RWI group. This comparison showed a significant difference between the two groups $(P<0.01$, Figure 2C).

Effect of muscimol microinjection into the $\mathrm{FN}$ on the discharge frequency of the GSN

To further clarify the role of the peripheral sympathetic pathway in the effect of muscimol microinjection into the FN, the discharge frequency of the GSN was recorded in rats prior to and following the microinjection of muscimol into the FN. Three discharge frequencies were chosen from three different time points, as shown in Figure 3A. The computations and analyses revealed that the GSN mean discharge frequency showed an upward trend from $396 \pm 12.6 \mathrm{~Hz}$ (before the microinjection) to $1082 \pm 36.1 \mathrm{~Hz}$ (after the microinjection) $(P<0.01$, $n=6)$.

A
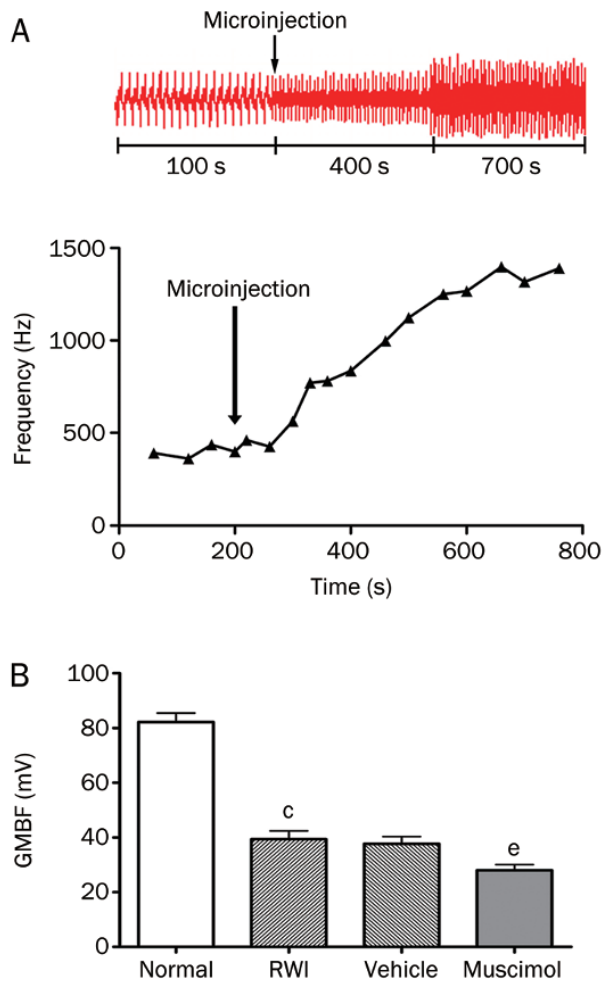

Figure 3. (A) Muscimol microinjection into FN on discharge frequency of GSN. (B) Muscimol microinjection into FN on gastric mucosal blood flow. Normal: normal; RWI: restraint and water $\left(21 \pm 1^{\circ} \mathrm{C}\right)$-immersion, only; Vehicle: microinjection of Vehicle into $\mathrm{FN}+\mathrm{RWI}$; Muscimol: muscimol was microinjected into the FN+RWI. Mean \pm SEM. $n=6$. ${ }^{c} P<0.01$ vs Normal group. ${ }^{e} P<0.05$ vs Vehicle group.

Effect of muscimol microinjection into the $\mathrm{FN}$ on the gastric mucosal blood flow

Comparison of the gastric mucosal blood flow between the
Normal $(82.17 \pm 3.32 \mathrm{mV})$ and RWI $(39.33 \pm 3.07 \mathrm{mV})$ groups indicated that restraint, cold water and immersion caused an obvious decrease in the gastric mucosal blood flow assessed by the Laser-Doppler flowmeter. The blood flow was $37.67 \pm 2.67 \mathrm{mV}$ in the Vehicle group, which was not different from that of the RWI group. However, the blood flow was significantly lower, $28.00 \pm 2.08 \mathrm{mV}$, in the muscimol group. There was a significant difference between the muscimol and Vehicle groups $(P<0.05$, Figure $3 \mathrm{~B})$.

Effect of muscimol microinjection into the FN on the proliferation of gastric mucosa cells in stress-induced gastric mucosal damage

As indicated in Figure 4A and 4B, PCNA-positive cells (ie, proliferating cells) had brown-stained nuclei. Although there was an abundance of proliferating cells in the Normal group $(55.17 \% \pm 6.05 \%)$, the percentage of proliferating cells was lower, $23.00 \% \pm 2.63 \%$, in the Vehicle group. In the muscimol group, it was significantly lower than that in the Vehicle group $(9.83 \% \pm 1.93 \%, P<0.01)$.

Effect of muscimol microinjection into the FN on the apoptosis of gastric mucosal cells in stress-induced gastric mucosal damage Figure 4C and 4D shows that the average percentage of apoptotic cells was approximately $2.33 \% \pm 0.90 \%$ in the Normal group. In the Vehicle group, the percentage of apoptotic cells was significantly higher $(35.67 \% \pm 4.66 \%, P<0.01)$ than that in the Normal group. In the muscimol group compared with the Vehicle group, we observed an increase in the percentage of cells undergoing apoptosis $(66.50 \% \pm 4.91 \%, P<0.01)$.

Effect of muscimol microinjection into the FN on the expressions of Caspase-3, Bax, and $\mathrm{Bcl}-2$ by the gastric mucosa in stressinduced gastric mucosal damage

Compared with the Normal group, the expression of Bax and Caspase- 3 was higher in the RWI group, but that of Bcl-2 was lower (Figure $5 ; P<0.05$ ). The increased expression of Bax and Caspase- 3 and the decreased expression of Bcl-2 were also observed in the muscimol group, but at levels that differed from those in the RWI group $(P<0.05)$.

Effect of muscimol microinjection into the FN on the MDA content and SOD activity in stress-induced gastric mucosal damage

The MDA content of the gastric mucosa was higher in the RWI $(7.31 \pm 0.60 \mathrm{nmol} / \mathrm{mg})$ and Vehicle $(6.72 \pm 0.65 \mathrm{nmol} / \mathrm{mg})$ groups than that in the Normal group $(4.69 \pm 0.30 \mathrm{nmol} / \mathrm{mg})$. The MDA content was markedly higher in the muscimol group $(12.32 \pm 1.47 \mathrm{nmol} / \mathrm{mg}, P<0.05)$ than that in the Vehicle group (Figure 6A). The SOD activity in the Normal group remained at a high level $(98.33 \pm 6.35 \mathrm{U} / \mathrm{mg})$. In contrast, it was lower, $64.17 \pm 8.41 \mathrm{U} / \mathrm{mg}$, in the RWI group and the Vehicle group, $60.50 \pm 8.98 \mathrm{U} / \mathrm{mg}$. Compared with the Vehicle group, the SOD activity in the muscimol group was significantly lower (24.83 $\pm 5.46 \mathrm{U} / \mathrm{mg}, P<0.05)$ (Figure 6B). 
A
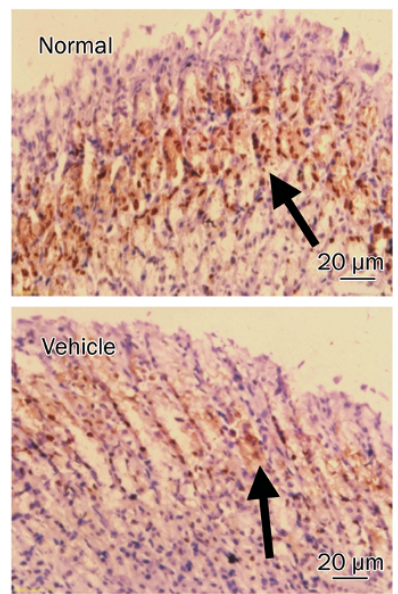
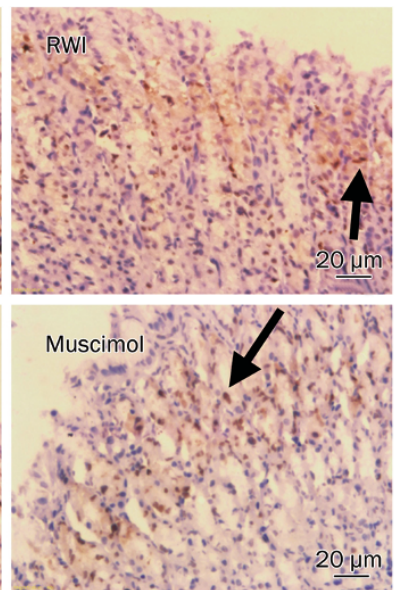

C
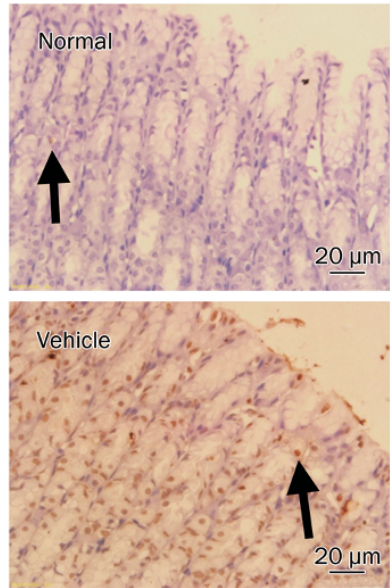
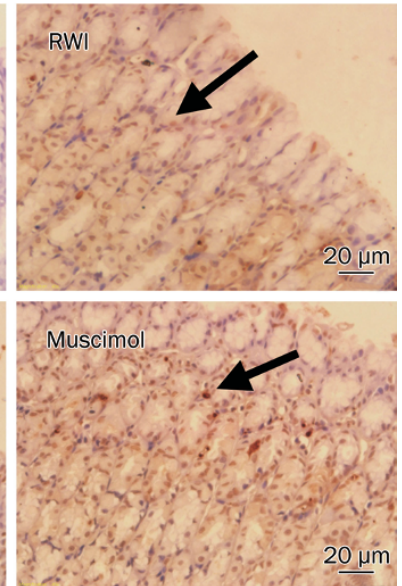

B

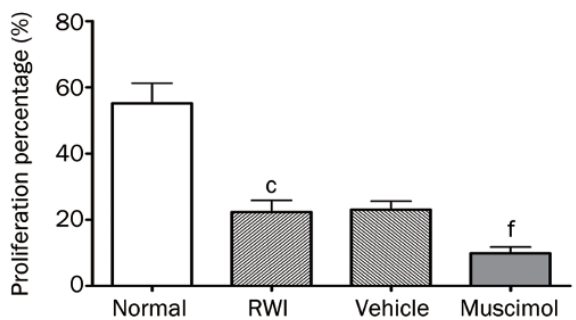

D

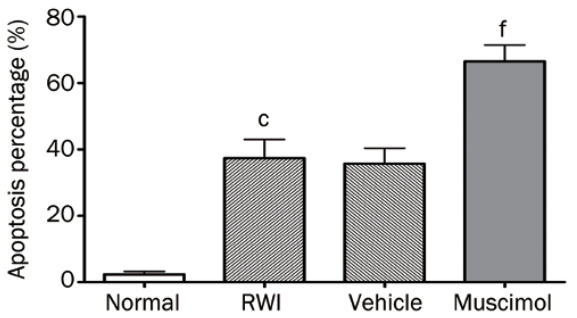

Figure 4. (A and B) Muscimol microinjection into FN on proliferation of gastric mucosal cells on stress-induced gastric mucosal damage in rats. The arrow areas are the positive cells. ( $C$ and D) Muscimol microinjection into FN on apoptosis of gastric mucosal cells on stress-induced gastric mucosal damage in rats. Normal: normal; RWI: restraint and water $\left(21 \pm 1^{\circ} \mathrm{C}\right)$-immersion, only; Vehicle: microinjection of Vehicle into FN+RWI; Muscimol: microinjection of muscimol into FN+RWI. Mean \pm SEM. $n=6$. ${ }^{\mathrm{c}} P<0.01$ vs Normal group. ${ }^{\mathrm{f}} P<0.01$ vs Vehicle group.
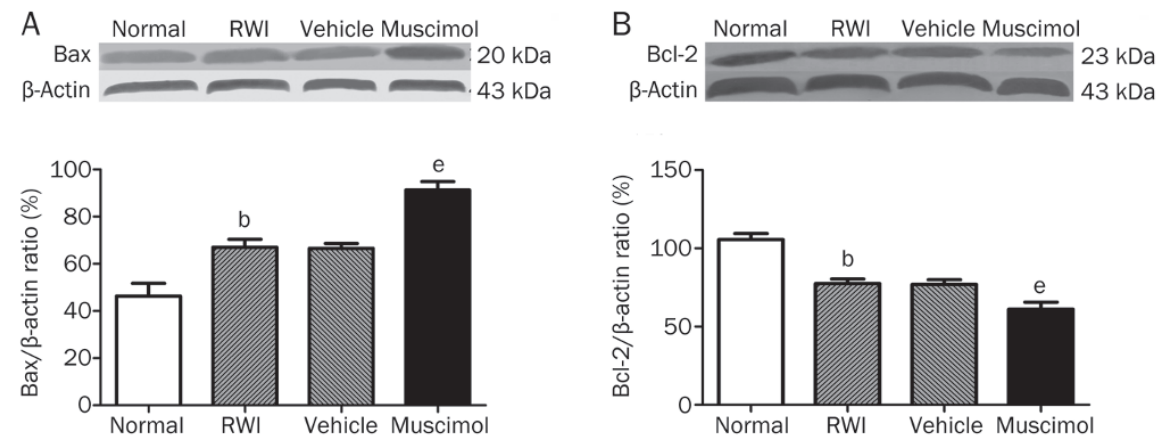

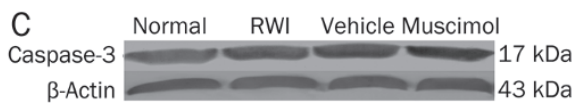

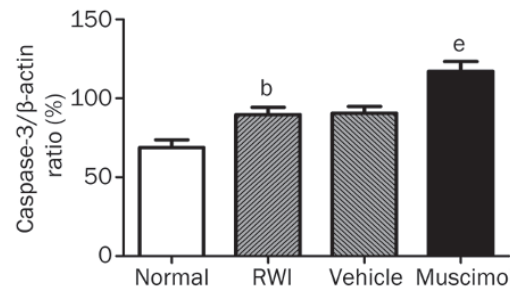

Figure 5. Muscimol microinjection into $\mathrm{FN}$ on expressions of $\mathrm{Bax}, \mathrm{Bcl}-2$, and Caspase-3 of gastric mucosal cells on stress-induced gastric mucosal

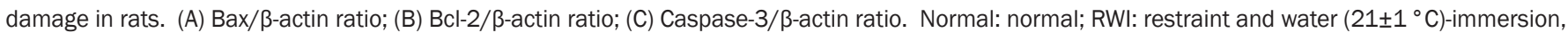
only; Vehicle: microinjection of Vehicle into FN+RWI; Muscimol: microinjection of muscimol into FN+RWI. Mean \pm SEM. $n=6$. ${ }^{\mathrm{b}} P<0.05$ vs Normal group. ${ }^{\mathrm{e}} \mathrm{P}<0.05$ vs Vehicle group.

\section{Effect of muscimol microinjection into the FN on the gastric juice volume and acidity}

As shown in Table 1, microinjection of muscimol resulted in a significantly higher gastric juice volume and acidity in the muscimol group than that in the Normal group. The microinjection of muscimol into the FN possibly stimulates the gastric acid secretory function, causing the intensifying effect on stress-induced gastric mucosal damage.

\section{Discussion}

The cerebellum is considered to be not only a traditional subcortical somatic motor center but also an essential component in the central integration of visceral activities ${ }^{[28,29]}$.

In our previous study, we inferred that the FN neuron, not the crossing fiber, was involved in the regulation of stressinduced gastric mucosal damage; however, the details of the underlying mechanisms were not clear. In this study using the microinjection of muscimol into the FN, we sought to 

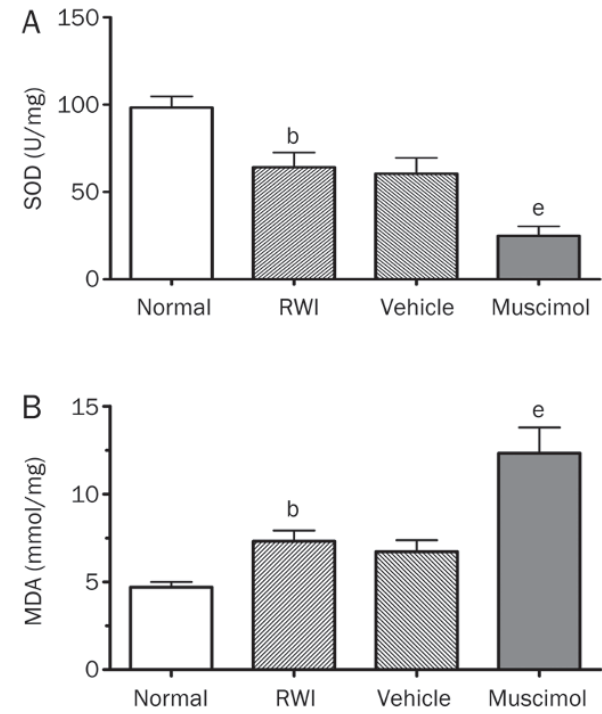

Figure 6. Muscimol microinjection into FN on MDA content and SOD activity on stress-induced gastric mucosal damage in rats. (A) MDA contents; (B) SOD activity. Normal: normal; RWI: restraint and water $\left(21 \pm 1^{\circ} \mathrm{C}\right)$-immersion, only; Vehicle: microinjection of Vehicle into $\mathrm{FN}+\mathrm{RWI}$; Muscimol: microinjection of muscimol into FN+RWI. Mean \pm SEM. $n=6$. ${ }^{\mathrm{b}} \mathrm{P}<0.05$ vs Normal group. ${ }^{\mathrm{e}} \mathrm{P}<0.05$ vs Vehicle group.

Table 1. Muscimol microinjection into $\mathrm{FN}$ on gastric juice volume and acidity in rats. Mean \pm SEM. ${ }^{\mathrm{b}} P<0.05$ vs Normal group.

\begin{tabular}{lcc}
\hline & Normal $(n=6)$ & Muscimol $(n=6)$ \\
\hline Volume $(\mathrm{mL})$ & $1.87 \pm 0.23$ & $3.77 \pm 0.37^{\mathrm{b}}$ \\
Acidity $\left(10^{-5} \mathrm{~mol} / \mathrm{mL}\right)$ & $4.51 \pm 0.31$ & $7.82 \pm 1.03^{\mathrm{b}}$ \\
\hline
\end{tabular}

reveal the association of the $\mathrm{GABA}_{\mathrm{A}}$ receptor in the $\mathrm{FN}$ with stress-induced gastric damage. The microinjection of different doses of muscimol into the FN exacerbated stress-induced gastric mucosal damage in a dose-dependent manner, whereas the microinjection of bicuculline, a $\mathrm{GABA}_{\mathrm{A}}$ receptor antagonist, attenuated this damage. Therefore, we assume that the $\mathrm{GABA}_{\mathrm{A}}$ receptor is one of the vital components in the mechanism by which the FN regulates stress-induced gastric damage.

In the study of cerebellar-hypothalamic circuits, the exacerbating effect of muscimol microinjection was cancelled by pretreatment with electrical lesions of the DSCP or by chemical ablation of the LHA. As reported in a previous study, electrical stimulation of the LHA decreased the gastric mucosal blood flow and produced exacerbated gastric ischemia-reperfusion injuries ${ }^{[15]}$. Consequently, we inferred that this effect may be directly mediated by LHA outflow, through its regulation of the gastric vasculature ${ }^{[30,31]}$. In this study, the discharge frequency of the GSN increased and the gastric mucosal blood flow decreased after microinjection of muscimol into the FN. In summary, we presumed that the microinjection of musci- mol into the FN increased the discharge of the GSN mediated by the LHA, resulting in gastric vasoconstriction of the submucosa and mucosa and a decrease in the mucosal blood flow, thereby exacerbating the stress-induced gastric mucosal damage.

Stress-induced gastric mucosal damage is a complex pathological process. Maintaining a dynamic balance between the epithelial cellular proliferation and apoptosis (programmed cell death) is essential for maintaining gastric mucosal integrity ${ }^{[16,32]}$. A wide range of studies have shown that stressinduced gastric ulcerations are accompanied by alterations in Bcl-2 and Bax ${ }^{[33]}$. Caspase-3 has also been identified as a key mediator of the apoptosis of mammalian cells ${ }^{[34]}$.

To clarify the cellular and molecular mechanism of the effects of muscimol microinjection into the FN on stressinduced gastric damage, the expression of $\mathrm{Bcl}-2$, Bax, and Caspase-3 proteins was assessed by Western blotting. Proliferation and apoptosis in the gastric mucosal epithelium were detected by performing SABC immunohistochemical staining and TUNEL staining, respectively. The microinjection of muscimol decreased the number of proliferative cells, increased the number of cells undergoing apoptosis, down-regulated Bcl-2 protein expression and up-regulated Bax and Caspase-3 protein expression during stress-induced gastric damage in rats. As a consequence, these results demonstrated that microinjection of muscimol into the FN effectively intensified stressinduced gastric damage via the promotion of gastric mucosal cellular apoptosis and the inhibition of gastric mucosal cellular proliferation in rats.

The levels of MDA and SOD are considered to be standards for the evaluation of the oxidative/anti-oxidative status of the gastric mucosa of rats. In this study, compared with the RWI group, the microinjection of muscimol decreased the SOD activity and increased the MDA content. Thus, the microinjection of muscimol into the FN decreased the anti-oxidant levels of the gastric mucosa injury induced by RWI.

A vast amount of data has shown that gastric acidity is a key contributor to stress-induced gastric mucosal damage induced by $\mathrm{RWI}^{[35]}$. In a previous study ${ }^{[36]}$, an altered ability of the hypothalamus was suggested to modulate gastric function, including the secretory and motor responses to stress. In agreement with that idea, we also detected alterations in gastric juice volume and acidity. Our results revealed that microinjection of muscimol into the FN significantly increased the gastric juice volume and acidity. We concluded that variation in the gastric gland secretory function is involved in the FN-mediated regulation of stress-induced gastric mucosal damage.

In conclusion, our study assessed the role of the FN in stressinduced gastric damage at the tissue, cellular and molecular levels. The study indicated that the cerebellar FN, an important area of the CNS, participates in the development of stressinduced gastric damage, through GABAergic neural pathways and the $\mathrm{GABA}_{\mathrm{A}}$ receptor. Cerebellar-hypothalamic circuits, especially those involving the DCSP and the LHA, participate in the FN-mediated regulation of the stress-induced gastric 
mucosal damage. The neuro-regulatory mechanism underlying our observations could be that microinjection of muscimol into the FN neurons, via their cerebellar-hypothalamic projections, arouses the activity of the GSN, reduces gastric mucosal blood flow and, finally, exacerbates stress-induced gastric damage. This stress-induced gastric damage is observed at the histological level, with muscimol injection leading to the inhibition of gastric mucosal cellular proliferation, the promotion of cellular apoptosis, a decrease in the anti-oxidant levels and an increase in gastric juice volume and acidity.

We believe that this research provides novel insight into the characteristics of the cerebellum with respect to regulating gastrointestinal function and into potential methods for treating gastrointestinal diseases.

\section{Abbreviations}

DSCP, decussation of superior cerebellar peduncle; FN, fastigial nucleus; GABA, gamma-aminobutyric acid; GMDI, gastric mucosal damage index; GSN, greater splanchnic nerve; LHA, lateral hypothalamic area; MDA, malondialdehyde; PCNA, proliferating cell nuclear antigen; RWI, restraint and water $\left(21 \pm 1^{\circ} \mathrm{C}\right)$-immersion; SOD, superoxide dismutase; TUNEL, transferase-mediated dUTP-biotin nick end-labeling.

\section{Acknowledgements}

This project was supported by grants from the National Natural Science Foundation of China (№ 30570671), the Educational Science Research Foundation of Jiangsu Province (No 10KJB310015), the Research Foundation of Xuzhou Medical College (Nos 07KJ58 and 07KJ34) and the Xuzhou Social Development Fund (№ XM08C062).

\section{Author contribution}

Jian-fu ZHANG and Su-juan FEI designed the research; Jinzhou ZHU performed the research and wrote the paper; and Sheng-ping ZHU, Zhang-bo LIU, Ting-ting LI, and Xiao QIAO analyzed the data.

\section{References}

1 Das D, Banerjee RK. Effect of stress on the antioxidant enzymes and gastric ulceration. Mol Cell Biochem 1993; 125: 115-25.

2 Konturek PK, Brzozowski T, Konturek SJ, Dembinski A. Role of epidermal growth factor, prostaglandin, and sulfhydryls in stressinduced gastric lesions. Gastroenterology 1990; 99: 1607-15.

3 Gao L, Fei S, Qiao W, Zhang J, Xing H, Du D. Protective effect of chemical stimulation of cerebellar fastigial nucleus on stress gastric mucosal injury in rats. Life Sci 2011; 88: 871-8.

4 Ottersen OP, Storm-Mathisen J. Glutamate- and GABA-containing neurons in the mouse and rat brain, as demonstrated with a new immunocytochemical technique. J Comp Neurol 1984; 229: 374-92.

5 Anchisi D, Scelfo B, Tempia F. Postsynaptic currents in deep cerebellar nuclei. J Neurophysiol 2001; 85: 323-31.

6 Mouginot D, Gahwiler BH. Characterization of synaptic connections between cortex and deep nuclei of the rat cerebellum in vitro. Neuroscience 1995; 64: 699-712.

7 Teune TM, van der Burg J, de Zeeuw Cl, Voogd J, Ruigrok TJ. Single Purkinje cell can innervate multiple classes of projection neurons in the cerebellar nuclei of the rat: a light microscopic and ultrastructural triple-tracer study in the rat. J Comp Neurol 1998; 392: 164-78.

8 Sangrey T, Jaeger D. Analysis of distinct short and prolonged components in rebound spiking of deep cerebellar nucleus neurons. Eur J Neurosci 2010; 32: 1646-57.

9 Chen S, Hillman DE. Colocalization of neurotransmitters in the deep cerebellar nuclei. J Neurocytol 1993; 22: 81-91.

10 Kawaguchi S, Ono T. Bicuculline and picrotoxin sensitive inhibition in interpositus neurones of cat. Brain Res 1973; 58: 260-5.

11 Wojtowicz JM, Marshall KC, Hendelman WJ. Electrophysiological and pharmacological studies of the inhibitory projection from the cerebellar cortex to the deep cerebellar nuclei in tissue culture. Neuroscience 1978; 3: 607-18.

12 Haines DE, Dietrichs E, Mihailoff GA, McDonald EF. The cerebellarhypothalamic axis: basic circuits and clinical observations. Int Rev Neurobiol 1997; 41: 83-107.

13 Jansen AS, Hoffman JL, Loewy AD. CNS sites involved in sympathetic and parasympathetic control of the pancreas: a viral tracing study. Brain Res 1997; 766: 29-38.

14 Zhang JF, Zhang YM, Yan CD, Zhou XP. Neuroregulative mechanism of hypothalamic paraventricular nucleus on gastric ischemia-reperfusion injury in rats. Life Sci 2002; 71: 1501-10.

15 Zhou XP, Zhang JF, Yan CD, Zhang YM. Effects of electrical stimulation of lateral hypothalamic area on gastric ischemia-reperfusion injury in rats. Sheng Li Xue Bao 2002; 54: 435-40.

16 Konturek PC, Brzozowski T, Konturek SJ, Pajdo R, Konturek JE, Kwiecien S, et al. Apoptosis in gastric mucosa with stress-induced gastric ulcers. J Physiol Pharmacol 1999; 50: 211-25.

17 Paxinos G, Watson C. The rat brain in stereotaxic coordinates. 6th ed. Amsterdam; Boston; Academic Press/Elsevier; 2007.

18 Kotz CM, Wang C, Teske JA, Thorpe AJ, Novak CM, Kiwaki K, et al. Orexin A mediation of time spent moving in rats: neural mechanisms. Neuroscience 2006; 142: 29-36.

19 Blake DJ, Gladfelter WE. Wheel-running activity after kainic acid injection into lateral hypothalamus of rats. Physiol Behav 1986; 36: 1009-16.

20 Nemeroff CB, Hernandez DE, Orlando RC, Prange AJ Jr. Cytoprotective effect of centrally administered neurotensin on stress-induced gastric ulcers. Am J Physiol 1982; 242: G342-6.

21 Du DS, Ma XB, Zhang JF, Zhou XY, Li Y, Zhang YM, et al. The protective effect of capsaicin receptor-mediated genistein postconditioning on gastric ischemia-reperfusion injury in rats. Dig Dis Sci 2010; 55: 3070-7.

22 Nakamura T, Kawabe K, Sapru HN. Cardiovascular responses to microinjections of urocortin 3 into the nucleus tractus solitarius of the rat. Am J Physiol Heart Circ Physiol 2009; 296: H325-32.

23 Lu JT, Zhao WD, He W, Wei W. Hedgehog signaling pathway mediates invasion and metastasis of hepatocellular carcinoma via ERK pathway. Acta Pharmacol Sin 2012; 33: 691-700.

24 Li HM, Wang YY, Wang HD, Cao WJ, Yu XH, Lu DX, et al. Berberine protects against lipopolysaccharide-induced intestinal injury in mice via alpha 2 adrenoceptor-independent mechanisms. Acta Pharmacol Sin 2011; 32: 1364-72.

25 Jiang W, Yang ZB, Zhou QH, Huan X, Wang L. Lipid metabolism disturbances and AMPK activation in prolonged propofol-sedated rabbits under mechanical ventilation. Acta Pharmacol Sin 2012; 33: $27-33$.

26 Du D, Ma X, Zhang J, Zhang Y, Zhou X, Li Y. Cellular and molecular mechanisms of 17 beta-estradiol postconditioning protection against gastric mucosal injury induced by ischemia/reperfusion in rats. Life Sci 2010; 86: 30-8. 
27 Corne SJ, Morrissey SM, Woods RJ. Proceedings: A method for the quantitative estimation of gastric barrier mucus. J Physiol 1974; 242 : 116P-117P.

28 Dietrichs E, Haines DE. Possible pathways for cerebellar modulation of autonomic responses: micturition. Scand J Urol Nephrol Suppl 2002; 210: 16-20.

29 Xu F, Frazier DT. Modulation of respiratory motor output by cerebellar deep nuclei in the rat. J Appl Physiol 2000; 89: 996-1004.

30 DiBona GF. Nervous kidney. Interaction between renal sympathetic nerves and the renin-angiotensin system in the control of renal function. Hypertension 2000; 36: 1083-8.

31 Dendorfer A, Thornagel A, Raasch W, Grisk O, Tempel K, Dominiak $P$. Angiotensin II induces catecholamine release by direct ganglionic excitation. Hypertension 2002; 40: 348-54.

32 Liu J, Li ZS, Wan XJ, Wang W. Expression and function of apoptosis- related genes $\mathrm{Bcl}-2 / \mathrm{Bax}$ and $\mathrm{Fas} / \mathrm{Fas} \mathrm{L}$ in the course of stress ulcer. Zhonghua Yi Xue Za Zhi 2003; 83: 504-9.

33 Liu MJ, Fei SJ, Qiao WL, Du DS, Zhang YM, Li Y, et al. The protective effect of 17 beta-estradiol postconditioning against hypoxia/reoxygenation injury in human gastric epithelial cells. Eur J Pharmacol 2010; 645: 151-7.

34 Kothakota S, Azuma T, Reinhard C, Klippel A, Tang J, Chu K, et al. Caspase-3-generated fragment of gelsolin: effector of morphological change in apoptosis. Science 1997; 278: 294-8.

35 Hayase M, Takeuchi K. Gastric acid secretion and lesion formation in rats under water-immersion stress. Dig Dis Sci 1986; 31: 166-71.

36 Banihashemi L, Rinaman L. Repeated brief postnatal maternal separation enhances hypothalamic gastric autonomic circuits in juvenile rats. Neuroscience 2010; 165: 265-77. 